\title{
Stenotic lesions
} Bengt Wranne, Helmut Baumgartner, Frank Flachskampf, Michael Hasenkam,
Fausto Pinto
Departments of

Clinical Physiology,

Linköping Heart

Centre, University

Hospital, Linköping,

Sweden

B Wranne

Department of

Cardiology, Vienna

General Hospital,

University of Vienna,

Vienna, Austria

H Baumgartner

Deparment of

Cardiology, RWTH

Aachen, Germany

F Flachskampf

Department of

Thoracic and

Cardiovascular

Surgery, Skejby

Sygehus, Aarhus

University Hospital,

Aarhus, Denmark

$M$ Hasenkam

University Hospital

Sta Maria, Clinica

Medica, Lisboa,

Portugal

F Pinto

All authors are member of the INVADYN group, a

concerted action in the

European Biomed Research programme.

Correspondence to:

Dr Bengt Wranne, Linköpin

Heart Centre, University

Hospital, Linköping,

Sweden.
A stenotic lesion becomes clinically significant when the vascular bed downstream from the stenosis is insufficiently supplied or the upstream vascular bed is subjected to intolerable stasis. The severity of the stenosis can be assessed by the extent of the symptoms of stasis or insufficient blood supply. From the point of view of fluid mechanics or haemodynamics, stenosis can be clinically assessed by the stenotic orifice area, the flow passing through the stenosis, and the driving pressure needed to expel the blood through the stenosis. Regardless of the increasingly sophisticated methods available for clinical assessment of stenotic lesions it is still important to be aware of these fundamental haemodynamic relations.

From the patient's point of view, accurate non-invasive methods for obtaining imaging and haemodynamic data are the most attractive. Therefore, Doppler echocardiography has had a considerable clinical impact during the past decade. Its use to assess stenotic lesions has expanded to the entire cardiovascular system and to the acquisition of many variables.

This paper deals with aortic and mitral stenoses and focuses on the clinical impact of Doppler echocardiography. The principles of fluid dynamics that apply to the aortic and mitral valves also apply to the tricuspid and pulmonary valves but these valves are not dealt with in this paper.

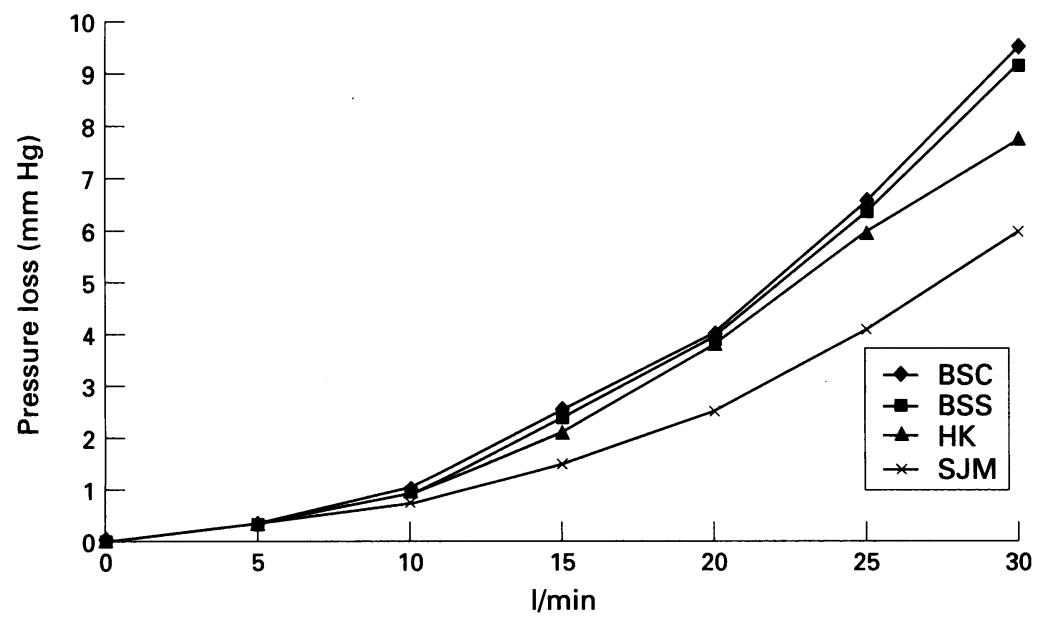

Figure 1 Relation between transvalvar pressure drop and transvalvar flow in a stenotic flow model. Four mechanical heart valves (all $27 \mathrm{~mm}$ aortic valves) were mounted in a flow loop: $B S C=$ Björk-Shiley convex/concave; BSS = Björk-Shiley standard; HK = Hall-Kaster (Medtronic-Hall); S丹M = St fude Medical. The pressure drop increased

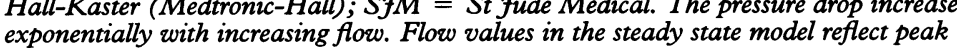
systolic conditions. (Adapted with permission from the J Biomech 1987;20:353-64. ${ }^{\text {) }}$ )

\section{Fluid dynamics}

One of the fundamental parameters describing the severity of a stenosis with turbulent flow is the haemodynamic resistance $(\mathrm{R})$

$$
\mathrm{R}=\Delta \mathrm{p} \div \mathrm{Q}^{2}
$$

where $\Delta \mathrm{p}$ is the transvalvar pressure drop and $\mathrm{Q}$ is the transvalvar flow. In this paper the expression "pressure gradient" is intentionally avoided and pressure loss, pressure drop, or pressure difference are instead used.The pressure gradient is $\mathrm{dp} / \mathrm{dx}$ (change in pressure as a function of change in distance). This is not measured either in clinical practice or in the experimental laboratory. What we measure is the pressure difference across a stenosis-in the cardiovascular system this will always be a pressure loss. There is an exponential increase in the transvalvar pressure drop as transvalvar flow increases (fig 1).

In many papers resistance is calculated by assuming a linear relation between pressure loss and flow $(\Delta \mathrm{p} / \mathrm{Q}) \cdot{ }^{2-4}$ From a fluid dynamic point of view this is incorrect because in important stenotic lesions flow is always turbulent. Despite this limitation the valvar resistance parameter calculated in this way gives information about the severity of a stenosis when invasive techniques have been used. In a strictly controlled in vitro experiment, however, Voelker $e t a l^{2}$ recently showed that valve resistance and stroke work loss, another proposed measure of stenosis severity, both vary considerably with flow. These measures should therefore be used with caution.

Ideally one catheter should be placed on each side of the valve to obtain simultaneous pressure recordings. When the pull-back technique is used beat-to-beat fluctuations in pressure will influence the pressure difference making measurements less reliable. Improper dampening caused by the fluid in the catheters and the poststenotic pressure recovery phenomenon ${ }^{5-6}$ are additional potential sources of errors.

As suggested by Hatle $e t a l^{7}$ the simplified Bernoulli equation can be used to assess the pressure difference non-invasively by Doppler ultrasound:

$$
\Delta \mathrm{p}=4 \times \mathrm{v}^{2}
$$

where $\mathrm{v}$ is the peak systolic blood velocity in the stenotic jet. The pressure difference estimated in this way is an instantaneous pressure drop, unlike the catheter derived pressure difference which usually is reported as a "peak to peak" pressure difference. The mean pressure difference is a more meaningful measure for 
comparing pressure drops estimated by the catheter and Doppler techniques since one can be calculated from the other. The Doppler derived mean pressure loss cannot be calculated directly from the mean velocity. It has to be calculated as the time average of the instantaneous pressure differences obtained from the Bernoulli relation. This is quite cumbersome to do manually, whereas with modern equipment this value is calculated at the same time as the velocity integral.

Cardiologists are conservative and most still use pressure differences calculated from Doppler data by the simplified Bernoulli equation. None the less it is more straightforward to calculate the time velocity integral, which conveys the same information as the mean pressure difference. Has the time come to report measured velocity data instead of derived pressure data? The advantage of using velocity data is obvious when the difference between Doppler-derived and catheter-measured pressure differences seen in some mechanical valve prosthesis is considered. ${ }^{6}$

Because the blood velocity in stenotic jets is usually high, continuous wave Doppler has to be used. This implies that velocity recordings are made in the entire cross field of the emitted and received ultrasound wavefronts. The reliability of this technique depends on its ability to detect the highest blood velocity area in the entire flow field and often a stand alone pencil probe has to be used. Furthermore, it requires the insonated ultrasound beam and the jet to be aligned. It is unwise to apply angle correction because the stenotic valve may direct the jet in any direction into the receiving chamber, ${ }^{8}$ increasing the problem of aligning the ultrasound wave and the jet.

Cardiac output, $Q$ can be assessed noninvasively from the simple equation:

$$
\mathrm{Q}=\overline{\mathbf{v}} \times \mathrm{A}
$$

where $\overline{\mathrm{v}}$ is the spatial mean blood velocity in the cross sectional area (A). Cardiac output can then theoretically be measured by multiplying area obtained from the cross sectional echocardiographic image and Doppler derived mean blood velocity. This technique has been suggested for use in the pulmonary artery, the mitral annulus, and the left ventricular outflow tract. All these techniques assume a flat blood velocity profile but this is not true of the pulmonary artery ${ }^{9}$ or the mitral annulus. ${ }^{1011}$ The technique, however, seems to work in the outflow tract of the left ventricle in patients with aortic stenosis. ${ }^{12}$ Rebreathing techniques have

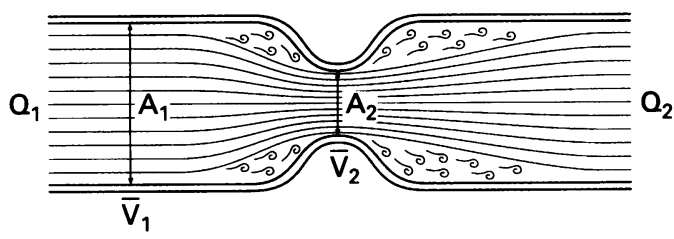

Figure 2 Illustration of the continuity equation. Volume flows on each side of a stenosis are identical. The reduced area in the stenosis necessitates a higher mean velocity to expel the same volume of fluid through the stenosis per time unit. also been applied for non-invasive determinations of cardiac output. ${ }^{13}$ Clearly both ultrasound and catheter based methods have sources of errors which the user must be aware of.

The functional area of the valve orifice can be estimated by the continuity equation, which states that volume flow at each crosssection is constant (fig 2). This is an expression of the conservation of mass principle. Using equation 3 :

$$
A_{1} \times \bar{v}_{1}=A_{2} \times \bar{v}_{2}
$$

where $A_{1}$ and $A_{2}$ are prestenotic and stenotic cross sectional areas, respectively, and $v_{1}$ and $v_{2}$ are the spatial and temporal mean velocities in the same locations. By planimetry of $A_{1}$ and measuring $\bar{v}_{1}$ and $\bar{v}_{2}$ the stenosis area can be calculated by rearranging equation (4):

$$
\mathrm{A}_{1}=\mathrm{A}_{1} \times \frac{\overline{\mathrm{v}}_{1}}{\overline{\mathrm{v}}_{2}} \text { or } \frac{\mathrm{A}_{1} \times \mathrm{TVI}_{1}}{\mathrm{TVI}_{2}}
$$

where $\mathrm{TVI}_{1}$ and $\mathrm{TVI}_{2}$ are the time velocity integrals of the prestenotic and stenotic areas, respectively.

The Gorlin formula ${ }^{14}$ is a special version of the continuity equation where the Bernoulli equation has been used to recalculate pressure as velocity. Accordingly, the Gorlin formula is used to estimate stenosis orifice area (A) from the transstenotic flow and the resulting transstenotic pressure drop. The original Gorlin formula has been challenged often-by in vitro studies, ${ }^{2}$ by animal experimental studies, ${ }^{15}$ and in humans. ${ }^{16}$ For example in the modification of the Gorlin formula used to assess aortic stenosis:

$$
\mathrm{A}=\frac{\mathrm{Qsm}}{\mathrm{k} \sqrt{\Delta \mathrm{p}_{\mathrm{sm}}}}
$$

where $\mathrm{Qsm}=$ mean systolic flow and $\Delta \mathrm{p}_{\mathrm{sm}}=$ the mean systolic transstenotic pressure drop; the factor " $k$ " was originally reported as $44 \cdot 3$ but values from 50 to 20 have been suggested. ${ }^{24}$

When "Gorlin areas" are compared with "continuity areas" it is important to remember that the Gorlin equation gives the anatomical area whereas the continuity equation calculates the flow area (which is smaller).

Stenoses always cause stenotic jets which include blood velocities above the critical value for causing turbulence. Turbulence can be heard with a stethoscope or recorded by an accelerometer. Turbulence can also be measured by pulsed and colour Doppler techniques. These require specialised equipment ${ }^{17} 18$ that is not used routinely.

\section{Aortic stenosis}

Aortic valve stenosis is often diagnosed before the patient is investigated with Doppler echocardiography. The cross sectional image will confirm this, but for a more exact assessment of the severity of disease Doppler recordings have to be made. The technique is well described elsewhere. ${ }^{19} \mathrm{We}$ only want to emphasise that the aortic valve must be 
Figure 3 Example of aortic stenosis of different severity. (A) $A$ case of mild stenosis with low velocity and an early maximum. (B) $A$ recording from a prosthetic St fude valve (size $19 \mathrm{~mm}$ ) in the aortic position. Note the early maximum despite the high velocity. This valve has no abnormal obstruction. (C) $A$ case with severe stenosis and a symmetrical curve. The patient has atrial fibrillation. Note the similarity in configuration despite the variations in velocity caused by differences in volume flow between the beats.
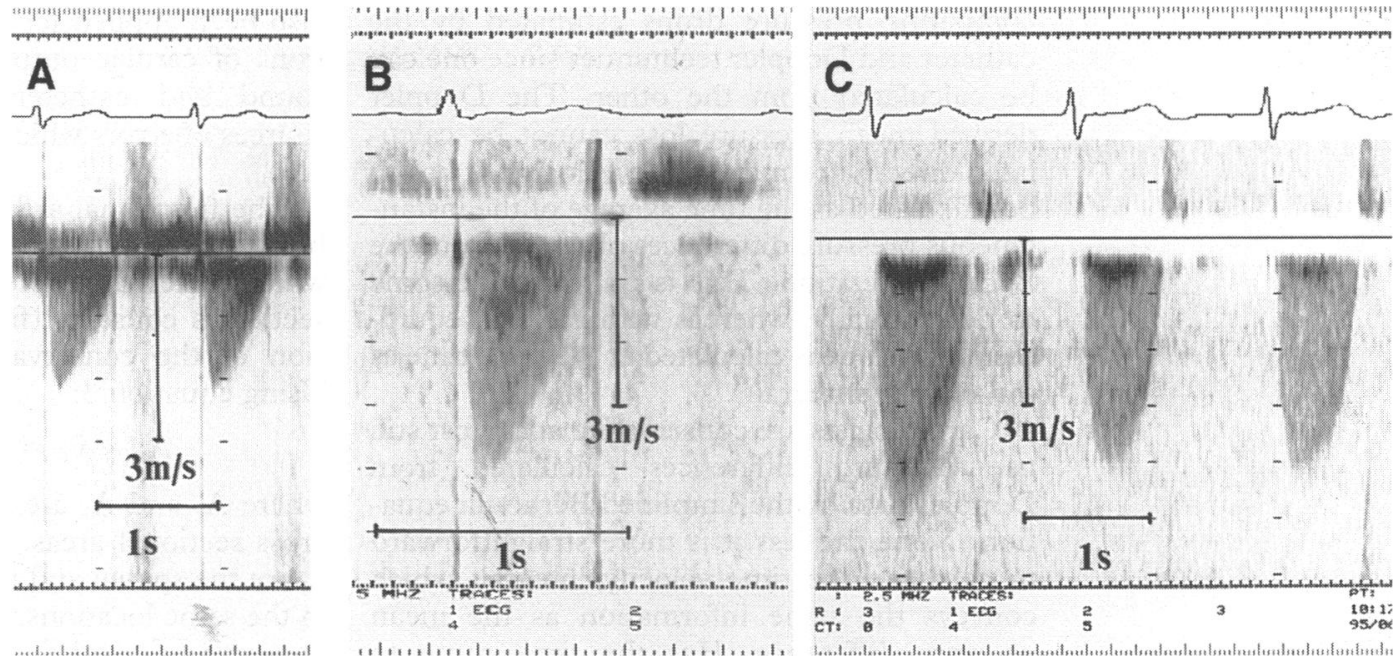

explored from several different positions to detect the highest velocities. None the less, velocities are flow dependent and may be high with fairly mild aortic stenosis or low with severe stenosis. Calculation of the stenotic area using the continuity equation, as described above, is then often of great help. This is achieved by measuring the velocity in the stenosis and in the outflow tract of the left ventricle and multiplying it by the subvalvar area obtained from the cross sectional image. In most cases the subvalvar flow profile is flat. ${ }^{12}$ However, since the subvalvar diameter is sometimes difficult to measure this approach is not always feasible. Visual analysis of the spectral curve is always important. With mild stenosis and high flow, the peak velocity occurs early in systole, whereas with severe stenosis the maximum is reached in midsystole - that is, the curve is symmetrical (fig 3).

Analysis of the spectral curve is also of special importance when subvalvar obstructions are assessed. Dynamic subvalvar obstruction, such as that seen in hypertrophic obstructive cardiomyopathy, typically produces a spectral curve with late maximum (fig 4) in addition to well known signs such as septal hypertrophy, systolic anterior motion of the mitral valve, and premature closure of the aortic valves. If a patient with dynamic subvalvar obstruction also has mitral regurgitation this can start late and peak late and may be mixed with the sub- valvar component.

Patients with aortic valve stenosis sometimes also have dynamic subvalvar obstructions. This is of special importance because surgical resection of the hypertrophic septum may be necessary as part of the surgical procedure. The information is also relevant to the treatment of the patient in the immediate postoperative period when inotropic drugs, which may provoke dynamic outflow obstruction, must be used with care.

Patients with aortic stenosis who are in cardiac failure are often difficult to assess because they have a low cardiac output and therefore low blood velocities and accordingly a low transvalvar pressure loss. In addition to visual analysis of the spectral curve, measurement of ejection time is of value here. A normal ejection time in a patient with cardiac failure is highly indicative of severe aortic stenosis or regurgitation..$^{20}$

Use of the continuity equation in low flow states may yield falsely low valve areas. Dobutamine ${ }^{22-24}$ or exercise echocardiography ${ }^{25}$ have been suggested as being helpful in these circumstances. The rationale behind this is that if the valve area increases with increasing flow, then the stenosis can not be severe. It is not obvious from these reports that this is the final answer to this question.

Transoesophageal echocardiography with its higher resolution and overall image quality
Figure 4 Spectral Doppler recordings from left ventricular outflow in a patient with obstructive cardiomyopathy. Note how difficult it can be to record the highest velocities, the late peak when these high velocities are recorded, and how the appearance of the spectral curve changes when these high velocities are missed.

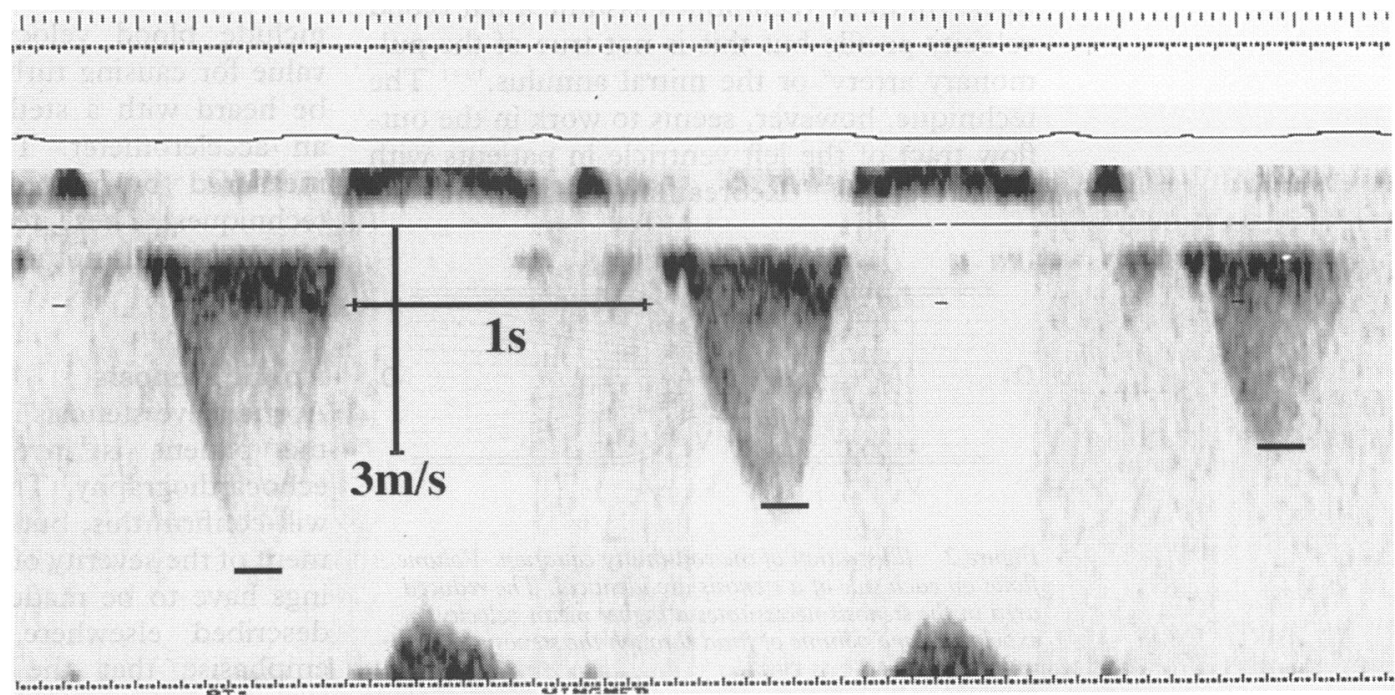




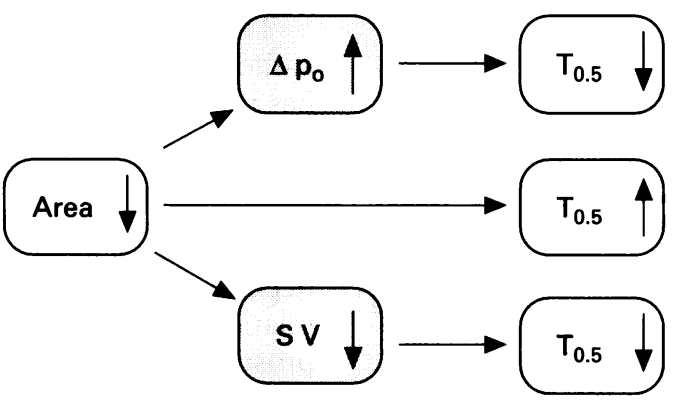

Figure 5 Shaded boxes show haemodynamic conditions known to occur with increasing severity of mitral stenosisthat is, increasing pressure difference $\left(\Delta p_{0}\right)$ across the valve and decreasing stroke volume (SV). The influence on pressure half time $\left(T_{0.5}\right)$ is shown to the right. Thus the direct and net effect of decreasing area is a prolonged pressure half time, but the extent of change depends on changes in $\Delta p_{o}$ and $S V$. The expected changes in both tend to shorten $T_{0.5 .}$ Reproduced with permission from the $\mathrm{J} \mathrm{Am}$ Soc Echocardiogr 1988;1:313-21.33

allows direct visualisation of the aortic orifice area. Planimetry of aortic valve area using multiplane transoesophageal transducers has been described. The level of the smallest orifice in the long axis view $\left(130-150^{\circ}\right)$ is located and the transducer plane is then rotated to a short axis view at $40-60^{\circ}$. Planimetry is performed in this view. Sometimes colour Doppler can be helpful to delineate the orifice. Sometimes planimetry is not feasible when there is heavy calcification, and misalignment with the valve plane may also induce errors. Good correlations with Gorlin areas have, however, been reported. ${ }^{26}$ This technique should be reserved for the few patients in whom conventional Doppler echocardiography does not give adequate information.

Doppler echocardiography, used in the way described above, generally provides an accurate assessment of the severity of stenosis. However, it is important that the assessment includes other information obtained in the echocardiographic investigation-for example, left ventricular cavity size and wall thickness.

\section{Mitral stenosis}

Diagnosis of mitral stenosis was the first clinical application of echocardiography. ${ }^{27}$ The $M$ mode two dimensional patterns are characteristic but do not provide exact information on the degree of stenosis. Doppler too was first used clinically to acquire data on mitral stenosis. $^{28}$

Planimetry of the mitral valve area from the cross sectional image is a well established technique ${ }^{29}$ but is subject to certain limitations: the stenotic orifice is seldom circular or even symmetrical, it may vary during the cardiac cycle. It may also vary with changing blood flow through the valve and be influenced by machine settings. Furthermore the technique requires that a directly orthogonal imaging plane can be achieved and recognition that excessive reflecting material, such as calcified tissue, may cause blooming-especially when older equipment is used. Those who see only occasional patients with mitral stenosis should use the technique with caution.

The pressure gradient half time method for assessing mitral stenosis was introduced for use at catheterisation ${ }^{30}$ and later adapted to echocardiography. ${ }^{31}$ It was initially applied with great enthusiasm and a number of reports supported its value. However, theory and model experiments predicted that the gradient half time should be influenced by atrial and ventricular compliance as well as the impedance exerted by the mitral orifice. ${ }^{32}$ Fluid dynamics predict that the gradient half time will be influenced by the diastolic transmitral flow and peak early transmitral pressure difference as well as the orifice area ${ }^{33}$ (fig 5).

When the half time concept was introduced and initially evaluated cardiologists were unaware of the importance of diastolic relaxation to mitral flow. We all now know that the flow velocity pattern of impaired diastolic relaxation can be characterised by a prolonged deceleration time (equivalent to a prolonged gradient half time). Therefore the combination of delayed ventricular relaxation and mild mitral stenosis may give a long gradient half time and simulate severe stenosis. ${ }^{34}$ It is also well known that gradient half time is incorrect after balloon dilatation of the mitral valve $e^{35}$ and shortened in patients with coexisting aortic regurgitation. ${ }^{36}$ For these reasons gradient half time shall not be used in isolation to assess mitral stenosis.

How then should we assess the severity of mitral stenosis? The qualitative diagnosis is generally obtained directly from the cross sectional image or $M$ mode recording. The degree of calcification and involvement of the subvalvar apparatus are also obtained from the cross sectional image. The mean pressure loss or the diastolic velocity integral can easily be calculated from the spectral curve. The motion of the interventricular septum will provide further information; the more severe the stenosis the later the filling of the left ventricle in relation to the right and consequently the more severe the abnormality of the septal motion. Assessment of right ventricular systolic pressure from a tricuspid regurgitation provides further evidence. The valve area can be calculated by planimetry of the cross sectional image and also by using the continuity equation, where flow can be calculated from the left ventricular outflow (if there is no aortic regurgitation). The proximal acceleration technique has also been used for this purpose. ${ }^{1537}$ Use of a combination of these techniques should generally give the correct diagnosis.

\section{Prosthetic valves}

Normal prosthetic valves cause some degree of flow obstruction, sometimes resulting in a significant pressure loss. It is important to recognise that Doppler-derived flow velocities and pressure differences vary with valve type and size because of the variation in effective orifice areas. Therefore, valve type and size must be specified when Doppler data are interpreted. Even when these variables have been defined, the reported range of normal velocities and gradients for prosthetic valves varies widely, 
Figure 6 Plots of mean catheter gradient and distance from valve. (A) Gradient obtained when the catheter was pulled back through the central orifice of the $S t$ fude valve. (B) Gradients obtained for two sizes of $S t$ fude valves when the catheter was pulled back through either the central or the side orifice. (C)

Gradients as a function of distance from the valve ring for Hancock tissue valves. Reproduced with the permission of

Circulation.

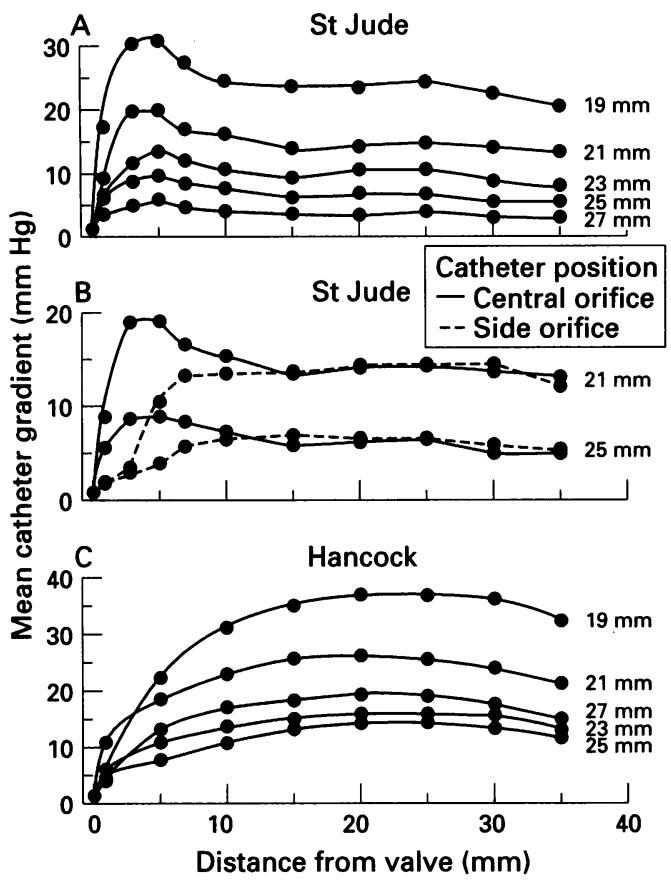

particularly for small valve sizes. ${ }^{38}$ The reason for this variation is the marked flow dependence of Doppler velocities across prosthetic valves. ${ }^{39}$ Therefore, particularly for small valve sizes, accurate evaluation of prosthetic valve function from Doppler velocities requires some information on the flow rate at which they were measured.

To provide a flow independent variable the continuity equation has been proposed as a method of calculating aortic prosthetic valve areas. However, at least for bileaflet valves, these calculations are not correct (see below). For mitral prosthetic valves, the pressure half time can be used. Normal values have been collected for most of the commonly used valves. ${ }^{38}$ Despite some variation, this variable has proved to be useful for the detection of prosthetic valve obstruction. As for the other variables, it is important to obtain baseline data early after surgery for comparison during later follow up. ${ }^{40}$ There are no data to justify the calculation of prosthetic valve areas from pressure half time.
The good agreement between Doppler and catheter data for native aortic valve stenosis has not always been found in heart valve prostheses. ${ }^{39}$ Particularly for mechanical valve prostheses, conflicting results have been reported. In vitro studies have helped to explain this phenomenon, demonstrating the dependence of the Doppler-catheter gradient relation on the geometric design of the valve prostheses. While acceptable agreement was found for normal Medtronic-Hall tilting disc and Hancock bioprosthetic valves, Doppler gradients significantly exceeded catheter gradients in normal bileaflet valves and caged ball valves. ${ }^{639}$ For bileaflet valves, it has also been shown that these discrepancies between Doppler and catheter gradients are not due to erroneous measurements by either technique but to spatial variation in pressure within and distal to the valve. Catheter pullback measurements (figs 6 and 7) showed that the Doppler calculated pressure loss accurately reflected the highest obtainable values obtained by the catheter technique. The maximal pressure loss was localised between the two leaflets of the valve and not in the side orifices. However, when distal pressures were measured $30 \mathrm{~mm}$ downstream from the valve, catheter gradients decreased and were significantly smaller than Doppler gradients (fig 7). The difference between Doppler and catheter measurements was, therefore, due to localised high gradients and pressure recovery and to the fact that the two techniques measured gradients in different locations; Doppler gradients reflect the highest gradient along the interrogation line and across the orifice (that is, the localised high gradient between the two leaflets) whereas catheterisation measures a recovered pressure further downstream from the valve. ${ }^{6}$

The discrepancies between Doppler and catheter pressure differences become particularly clinically relevant in small valves and at high flow rates, where differences as great as $44 \mathrm{mmHg}$ have been observed, ${ }^{39}$ but are of less importance in large valves with very low pressure differences. The relation between Doppler and catheter measurements is significantly altered by malfunction of the valve
Figure 7 Peak (left panel) and mean (right panel) Doppler derived gradients in relation to corresponding catheter gradients. Open circles refer to highest catheter gradient and closed circles to gradients $30 \mathrm{~mm}$ distal to the valve.

Measurements were obtained in St Fude valves (19-27 mm). Reproduced with the permission of Circulation. ${ }^{6}$

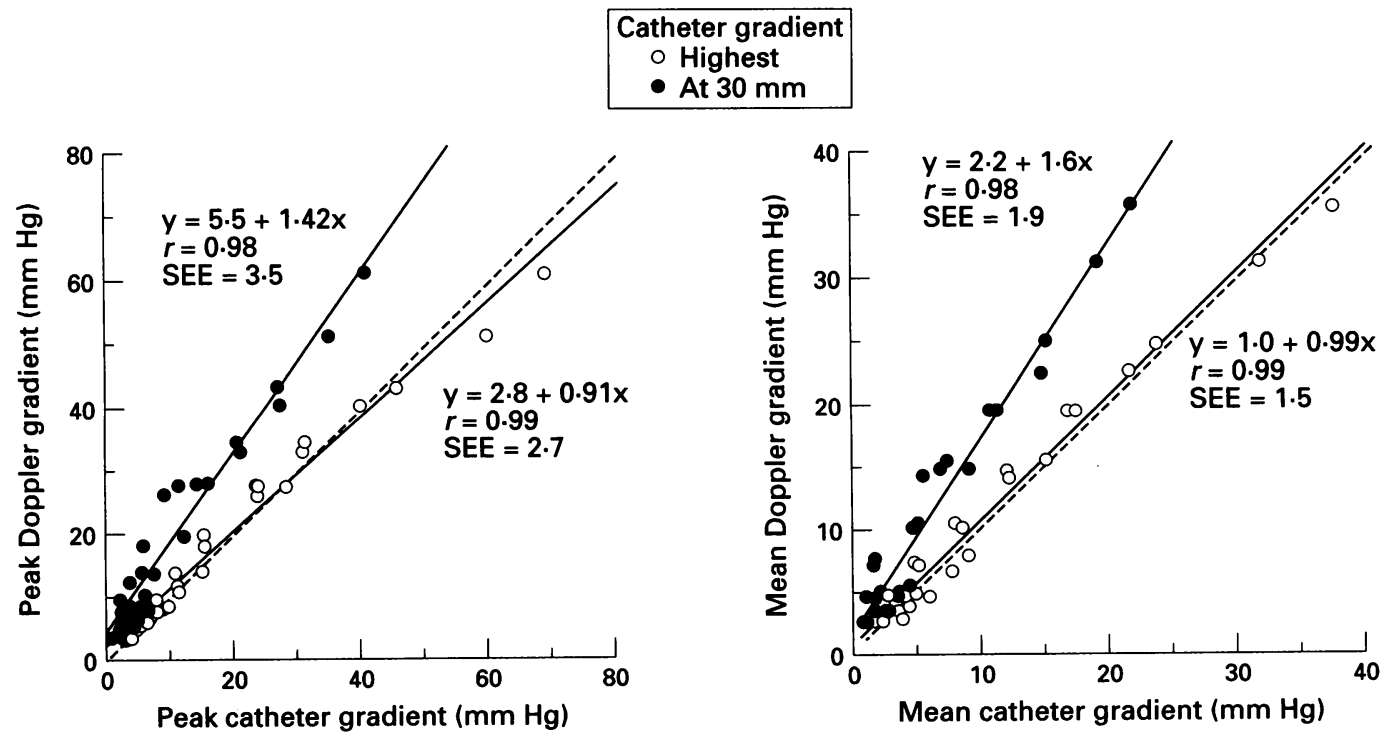


Figure 8 Correlation between mean Doppler and mean catheter gradients in a $19 \mathrm{~mm}$ CarboMedics bileaflet valve studied during normal function and in various stages of malfunction ranging from slightly restricted opening to total occlusion of one leaflet. The different symbols indicate the functional status at which the gradients were measured. Reproduced with the permission of Circulation. ${ }^{41}$

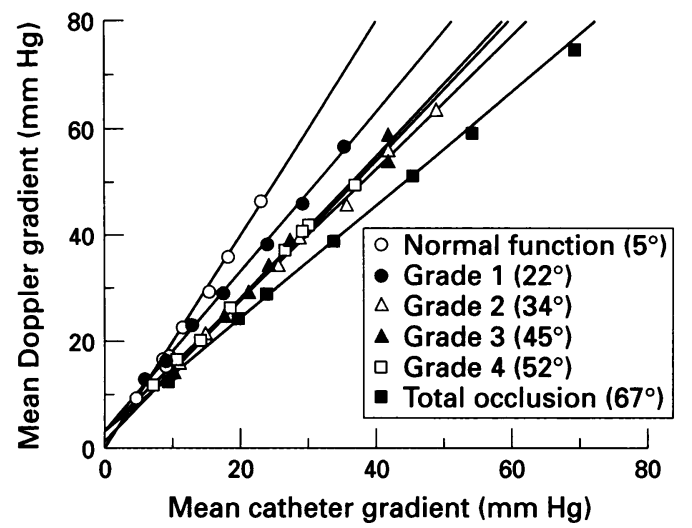

(fig 8). With increasing restriction of opening of one leaflet, the differences between Doppler and catheter gradients decrease and eventually almost disappear when one leaflet is totally occluded. This is due to the changing flow characteristics of this valve type ${ }^{41}$ that occur with malfunction. The pressure recovery seen in the "central tunnel" of normal bileaflet valves is not present with restricted leaflet motion where hydraulic conditions are similar to those seen in native stenotic valves.

These observations have several important implications. First, the dependence of the Doppler-catheter gradient relation on the functional status of the valve precludes the application of correcting factors. Second, one should not assess the function of bileaflet aortic valve prostheses by calculating the valve orifice area with the continuity equation. Because these calculations are based on localised high velocities between the two leaflets rather than on the average velocity across the orifice, the Doppler valve area underestimates the true effective orifice area of bileaflet valves. ${ }^{42}$ Since differences between localised high velocities and average velocities across the orifice decrease with increasing restriction of leaflet motion the decrease of Doppler valve area caused by restricted leaflet opening will also underestimate the true change in effective orifice area. Therefore, bileaflet valves with high Doppler gradients should be studied carefully with additional non-invasive techniques such as cinefluoroscopic evaluation of leaflet motion to avoid erroneous diagnoses of prosthetic valve stenosis. Transoesophageal echocardiography can often image leaflet motion in the mitral position very well.

In this review we have concentrated on aortic and mitral valve stenosis. The general principles described here for these valve lesions also apply to pulmonary and tricuspid stenosis. We believe that Doppler echocardiography is currently the best technique for evaluating valve lesions. However, a good knowledge of fluid dynamics, the machine, and clinical cardiology is needed to get optimal information from the investigation and to avoid mistakes in interpretation of data. Every echocardiogram has to be interpreted taking into account the clinical condition of the patient.

1 Hasenkam JM, Westphal D, Reul H, Gormsen J, Paulsen PK, Stødkilde-Jørgensen H. Three-dimensional visual- ization of axial velocity profiles downstream of six different mechanical aortic valve prostheses, measured with a hotfilm anemometer in a steady state flow model. $\mathcal{F}$ Biomech 1987;20:353-64

2 Voelker W, Reul H, Nienhaus G, Stelzer T, Schmitz B, Steegers A, et al. Comparison of valvular resistance, stroke work loss and Gorlin valve area for quantification of aortic stenosis. An in vitro study in a pulsatile aortic flow model. Circulation 1995;91:1196-204.

3 Ford LE, Feldman T, Chiu YC, Carroll JD. Hemodynamic resistance as a measure of functional impairment in aortic valvular stenosis. Circ Res 1990;66:1-7.

4 Cannon SR, Richards KL, Crawford M. Hydraulic estimation of stenotic orifice area: a correction of the Gorlin formula. Circulation 1985;71:1170-8.

5 Levine RA, Jimoh A, Cape EG, Mcmillan S, Yoganathan AP, Weyman A. Pressure recovery distal to a stenosis: potential cause of gradient "overestimation" by Doppler echocardiography. $¥ \mathrm{Am}$ Coll Cardiol 1989;13:706-13.

6 Baumgartner H, Khan S, DeRobertis M, Czer L, Maurer G. Discrepancies between doppler and catheter gradients in aortic prosthetic valves in vitro. A manifestation of localized gradients and pressure recovery. Circulation 1990;82:1467-75.

7 Hatle L, Angelsen BA, Tromsdal A. Non-invasive assessment of aortic stenosis by Doppler ultrasound. Br Heart $\mathcal{F}$ ment of aortic stenos

8 Grimes RY, Burleson A, Levine RA, Yoganathan AP. Quantification of cardiac jets: Theory and limitations. Quantification of cardiac jets: The

9 Sloth E, Pedersen EM, Nygaard H, Hasenkam JM, Juhl B. Multiplane transesophageal Doppler-echocardiography measurements of the velocity profile in the human pulmonary artery. $\mathcal{F} \mathrm{Am}$ Soc Echocardiogr 1994;7:132-40.

10 Kim WY, Bisgaard T, Nielsen SL, Poulsen JK, Pedersen EM, Hasenkam JM, et al. Two-dimensional mitral flow velocity profiles in pig models using epicardial echoDoppler-cardiography. $f$ Am Coll Cardiol 1994;24: 532-45.

11 Kupari M, Järvinen V, Poutanen V-P, Hekali P. Skewness of instantaneous mitral transannular flow-velocity profiles in normal humans. Am F Physiol 1995;268: H1232-38.

12 Janerot Sjöberg B, Loyd D, Ask P, Wranne B. Subaortic flow profiles in aortic valve disease. $\mathcal{F} \mathrm{Am}$ Soc Echocardiogr 1994;7:276-85.

13 Ohlsson J, Wranne B. Noninvasive assessment of valve area in patients with aortic stenosis. $7 \mathrm{Am}$ Coll Cardiol 1986; 7:501-8.

14 Gorlin R, Gorlin SG. Hydraulic formula for calculation of area of the stenotic mitral valve, other cardiac valves and central circulatory shunts. Am Heart $\mathcal{F}$ 1951;41:1-29.

15 Shiota T, Jones M, Valdes-Cruz LM, Shandas R, Yamada I, Sahn DJ. Color flow Doppler determination of transmitral flow and orifice area in mitral stenosis: Experimental evaluation of the proximal flow-convergence method. $\mathrm{Am}$ Heart $₹ 1995 ; 129: 114-23$.

16 Fischer JL, Haberer T, Dickson D, Henselmann L. Comparison of Doppler echocardiographic methods with heart catheterization in assessing aortic valve area in 100 heart catheterization in assessing aortic valve area in 100

17 Nygaard H, Paulsen PK, Hasenkam JM, Kromann-Hansen O, Pedersen EM, Rovsing PE. Quantitation of the turbulent stress distribution downstream of normal, diseased and artificial aortic valves in humans. Eur $₹$ Cardiothorac Surg 1992;6:609-17.

18 Nygaard $H$, Hasenkam JM, Pedersen EM, Kim WY, Paulsen PK. A new perivascular multielement pulsed Doppler ultrasound system for in vivo studies of velocity fields and turbulent stresses in large vessels. Med Biol Eng Comput 1994;32:55-62.

19 Skjaerpe T, Hegrenaes L, Hatle L. Noninvasive estimation of valve area in patients with aortic stenosis by Doppler ultrasound and two-dimensional echocardiography Circulation 1985;72:810-8.

20 Lewis RP, Rittgers SE, Forester WF, Boudoulas H. A critical review of the systolic time intervals. Circulation 1977; 56:146-58.

21 Nylander E, Ekman I, Marklund T, Sinnerstad B, Karlsson E, Wranne B. Severe aortic stenosis in elderly patients. Br Heart F 1986;55:480-7.

22 Burwash IG, Thomas DD, Sadahiro M, Pearlman AS, Verrier ED, Thomas R, et al. Dependence of Gorlin formula and continuity equation valve areas on transvalvular volume flow rate in valvular aortic stenosis. Circulation 1994;89:827-35.

23 Tardif J-C, Miller DS, Pandian NG, Schwartz SL, Gordon G, Tierney $R$, et al. Effects of variation in flow on aortic valve area in aortic stenosis based on in vivo planimetry of aortic valve area by multiplane transoesophageal of aortic valve area by multiplane transoes

24 deFilippi CR, Willet DL, Brickner E, Appleton CP, Yancy $\mathrm{CW}$, Eichhorn EJ, et al. Usefulness of Dobutamine $\mathrm{CW}$, Eichhorn EJ, et al. Usefulness of Dobutamine echocardiography in distinguishing severe from nonsevere valvular aortic stenosis in patients with depressed left ventricular function and

25 Burwash IG, Pearlman AS, Kraft CD, Miyake-Hull C, Healy NL, Otto CM. Flow dependence of measures of Healy NL, Otto CM. Flow dependence of measures of aortic stenosis severit

26 Hoffman R, Flachskampf FA, Hanrath P. Planimetry of orifice area in aortic stenosis using multiplane transorifice area in aortic stenosis using multiplane trans529-34. 
27 Edler I. Ultrasound-cardiogram in mitral valvular disease. Acta Chir Scand 1956;11:230-1.

28 Holen J, Aaslid R, Landmark K, Simonsen S. Determination of pressure gradient in mitral stenosis with a non invasive ultrasound Doppler technique. Acta Med Scand 1976;199:455-60

29 Henry WL, Griffith JM, Michaelis LL, McIntosh CL Morrow AG, Epstein SE. Measurement of mitral orifice area in patients with mitral valve disease by real-time two-dimensional echocardiography. Circulation 1975;51: $827-31$.

30 Libanoff AJ, Rodbard S. Evaluation of the severity of mitral stenosis and regurgitation. Circulation 1966;33:218-26.

31 Hatle L, Angelsen B, Tromsdal A. Noninvasive assessmen of atrioventricular pressure half-time by Doppler ultrasound. Circulation 1979;60:1096-104.

32 Thomas JD, Weyman AE. Doppler mitral pressure halftime: a clinical tool in search of theoretical justification. $f$ Am Coll Cardiol 1987;10:923-9.

33 Loyd D, Ask P, Wranne B. Pressure half-time does not always predict mitral valve area correctly. $f$ Am Soc Echocardiogr 1988;1:313-21.

34 Wranne B, Ask P, Loyd D. Analysis of different methods of assessing the stenotic mitral valve area with emphasis on the pressure gradient half-time concept. Am $f$ Cardiol 1990;66:614-20.

35 Thomas JD, Wilkins GT, Choong CYP, Abascal VM

Palacios IF, Block PC, et al. Inaccuracy of mitral pressure half-time immediately after percutaneous mitral valvotomy. Dependence on transmitral gradient and left atrial and ventricular compliance. Circulation 1988;78:980-93.

36 Flachskampf FA, Weyman AE, Gillam L, Liu CM, Abascal VM, Thomas JD. Aortic regurgitation shortens doppler pressure half-time in mitral stenosis: clinical evidence, in vitro simulation and theoretic analysis. $\mathrm{f} \mathrm{Am}$ Coll Cardio 1990;16:396-404.

37 Rodriguez L, Thomas JD, Monterosso V, Weyman AF Harrigan P, Mueller LN, et al. Validation of the proxima flow convergence method. Calculation of orifice area in patients with mitral stenosis. Circulation 1993;88: $1157-65$.

38 Reisner SA, Meltzer RS. Normal values of prosthetic valve Doppler echocardiographic parameters: a review. $f(A m$ Soc Echocardiogr 1988;1:201-10.

39 Baumgartner H, Khan S, deRobertis M, Czer L, Maurer G. Effect of prosthetic aortic valve design on the Doppler-catheter gradient correlation: an in vitro study of normal St Jude, Medtronic-Hall, Starr-Edwards and Hancock valves. F Am Coll Cardiol 1992;19:324-32.

40 Chambers J, Fraser A, Lawford P, Nihoyannopoulus, Simpson , Frer A, Lawford P, Ninoyannopoulus, Simpson IA. Echocardiographic assessment of artificial tion paper. Br Heart f 1994;71:6-14.

41 Baumgartner $\mathrm{H}$, Schima $\mathrm{H}$, Kühn $\mathrm{P}$. Effect of prosthetic valve malfunction on the Doppler-catheter gradient relavalve malfunction on the Doppler-catheter gradient rela-
tion for bileaflet aortic valve prostheses. Circulation 1993; 87:1320-7.

42 Baumgartner $\mathrm{H}$, Khan SS, deRobertis M, Czer LS, Maurer G. Doppler assessment of prosthetic valve orifice area. An in vitro study. Circulation 1992;85:2275-83. 